This is the peer reviewed version of the following article:

Morales-Narváez E., Merkoçi A.. Graphene Oxide as an Optical Biosensing Platform: A Progress Report. Advanced Materials, (2019). 31. 1805043: - . 10.1002/adma.201805043,

which has been published in final form at https://dx.doi.org/10.1002/adma.201805043. This article may be used for non-commercial purposes in accordance with Wiley Terms and Conditions for Use of Self-Archived Versions. 
DOI: $10.1002 /$ ((please add manuscript number))

Article type: Progress Report

\section{Graphene Oxide as an Optical Biosensing Platform - a Progress Report}

\section{Eden Morales-Narváez*and Arben Merkoçi*}

Prof. E. Morales-Narváez

Biophotonic Nanosensors Laboratory, Centro de Investigaciones en Óptica, A. C., Loma del Bosque 115, Lomas del Campestre, León, Guanajuato 37150, México

*E-mail: eden@cio.mx

Prof. A. Merkoçi

Nanobioelectronics and Biosensors Group, Catalan Institute of Nanoscience and Nanotechnology (ICN2) CSIC and BIST, Campus UAB, Bellaterra, 08193 Barcelona, Spain ICREA - Institució Catalana de Recerca i Estudis Avançats, Barcelona 08010, Spain *E-mail: arben.merkoci@icn2.cat

Keywords: 2D materials, bioanalytical applications, label-free biosensors, physicochemical properties, optical devices

Six years ago, crucial graphene oxide (GO) features such as the carbon/oxygen ratio, number of layers and lateral size were scarcely investigated and thus their impact on the overall optical biosensing performance was almost unknown. Nowadays valuable insights about these features are well documented in the literature, whereas others remain controversial. Moreover, most of the biosensing systems based on GO were amenable to operating as colloidal suspensions. Currently, the literature reports conceptually new approaches obviating the need of GO colloidal suspensions, enabling the integration of GO onto a solid phase and leading to their applications in new biosensing devices. Furthermore, most of the GO-based biosensing exploit photoluminescent signals. However, further progress has also been achieved in powerful label-free optical techniques exploiting GO in biosensing, particularly using optical fibers, surface plasmon resonance and surface enhanced Raman scattering. Herein, we offer a critical overview on these topics, highlighting the key role of the physicochemical properties of GO. We also highlight new challenges and opportunities within this exciting field. This Progress Report is a complementary approach of our previous article in Adv. Mater. 2012, 24, 3298, with special emphasis on recently discovered GO properties affecting biosensing behavior and innovative trends in optical biosensing based on GO.

\section{Introduction}

As a 2D material, graphene oxide (GO) is a lattice-like nanostructure of hexagonal carbon rings disrupted by oxygen containing-moieties. In 2012, we discussed the outstanding physicochemical properties offered by graphene oxide and how these features can be exploited in unprecedented optical biosensing systems. ${ }^{[1]}$ In fact, GO can be processed in suspension, easily complexed with biomolecules, and offers a universal highly efficient longrange photoluminescence quenching agent -among other functional properties. Furthermore, 
we highlighted that GO photoluminescences with energy transfer donor/acceptor molecules exposed in a planar surface. ${ }^{[1]}$ However, GO properties can be tailored according to its oxidation degree, lateral size and number of layers, which was something little explored 6 years ago, and thus their impact on the overall biosensing performance was almost unknown. In addition, most of the biosensing systems based on GO were amenable to working as colloidal suspensions. Though, recent literature reports conceptually new approaches obviating the need of colloidal suspensions, which facilitates integration of GO-based biosensors into the solid phase and allows for their applications in new devices. Furthermore, the majority of the GO-based optical biosensing techniques rely on photoluminescent signals. However, further progress has also been achieved in powerful label-free optical techniques exploiting GO in biosensing. Here, we introduce a progress report on this exciting topic, underscoring challenges and opportunities in (bio)sensing accordingly.

\section{The number of layers, lateral size, and oxidation degree of GO matter}

During a talk given by Prof. Mildred S. Dresselhaus in the International Conference Graphene 2012 at Brussels, she urged the scientific community to be careful with the specific properties of the utilized graphene derivatives. Prof. Dresselhaus raised awareness on the fact that the graphene derivatives studied in different parts of the world were not particularly the same, whereas they all -a family of materials- were just named graphene. Later (2013-2014), Prof. Alberto Bianco, Prof. Peter Wick and other members of the EU Graphene flagship project took action on this important matter and recommended a specific nomenclature and classification for 2D carbon materials, mainly based on their lateral size, number of layers and oxidation degree. ${ }^{[2,3]}$ Nowadays relevant insights about these features are well documented in the literature, whereas others remain little explored or controversial. The following section summarizes and highlights these crucial features with especial emphasis on their overall impact related to optical bio/sensing performance. 


\subsection{Number of layers}

GO aqueous suspensions are highly stable in the form of monolayers. However, GO multilayer films can be built via spin coating methods, layer-by-layer assembly or patterning through wax-printed membranes. ${ }^{[4-6]}$ Optical transparency may play a key role in visual detection, optoelectronic interfaces and wearable devices. In this context, light transmittance in GO can be controlled according to GO layers. In fact, GO optical transparency has been found to decrease with GO thickness, ${ }^{[4,7]}$ see Figure 1A. Hence, this feature should be carefully evaluated in GO-based bio/sensors intended for visual determination, optoelectronic interfaces and wearable devices. Moreover, GO thickness has been reported to be crucial in laser energy absorption capabilities and laser energy transfer for efficient laser desorption/ionization mass spectrometry analysis. ${ }^{\left[{ }^{8]}\right.}$ Interestingly, the optimum number of GO layers for this type of analysis is related to the chemical structures of small molecules. ${ }^{[8]}$ In addition, highly ordered layers of GO can be exploited as photonic crystals with potential applications in visually observable biosensing approaches via structural colors. ${ }^{[9]}$ Controversially, the mechanism of color reflection triggered by GO films has been analyzed using different methods which could be based on either, the interference between the reflections from the top and bottom surfaces of the GO coating on the macroscopic scale, ${ }^{[10]}$ or on the structure and the composition of multilayer GO at microscopic scale. ${ }^{[11]}$ Moreover, it is worth mentioning that the coating of silica photonic crystals with GO has been reported to modulate optical reflectance intensity and reflectance peak broadening, that is to say, GO is able to enhance antireflection and color strength properties, thus operating as a functional material for the manipulation of structural color, ${ }^{[12]}$ which might also be useful in relevant chemical sensing applications. ${ }^{[13]}$

It is well-known that GO can be exploited as an outstanding quencher of photoluminescence. ${ }^{[14]}$ To date, the literature offers more than 465 publications dealing with 
photoluminescent biosensing based on GO. Around 51\% of these publications report biosensing systems based on photoluminescence quenching (data provided via a Web of Science search). Although the number of layers of graphene-related materials has been reported to strongly modulate the photoluminescence quenching efficiency of these $2 \mathrm{D}$ materials, ${ }^{[15,16]}$ this feature is scarcely mentioned or considered by scientists and technologists working in this field. Interestingly, this phenomenon is still controversial as a theoretical and experimental approach claims that "the quenching efficiency increases with layer number", ${ }^{15]}$ see Figure 1B; whereas another experimental approach reports an opposite behavior -“the quenching factor (which represents the intensity of the studied quenching phenomenon) decreases from monolayer to 2-4 layers graphene", ${ }^{[16]}$ see Figure 1C. Moreover, to the best of our knowledge, focusing this relationship between layer number and quenching efficiency specifically on GO, this non-radiative energy transfer mechanism has not been systematically explored and reported. If action is taken to address this paucity, this might lead to new research and perhaps innovative applications. Regarding the effect of this feature on the overall performance of biosensing based on photoluminescence quenching, we believe that GO number of layers should be judiciously considered in optical biosensing systems based on the solid phase as this can strongly affect the overall analytical behavior of the studied biosensing systems, specifically in terms of limit of detection and dynamic range. Layer number has also been reported to tune photoluminescent properties of graphene oxide nanosheets. Actually, single layer graphene quantum dots have been reported to exhibit green photoluminescence and a quantum yield around $4 \%$, whereas multilayer graphene quantum dots show yellow photoluminescence and a quantum yield of $2.3 \%$ under the same excitation wavelength of $365 \mathrm{~nm}$, see Figure 1D. However, the lateral sizes of the studied materials were also different -15 and $18 \mathrm{~nm}$, respectively, ${ }^{[17]}$ which also dramatically affects photoluminescent GO properties as discussed below. Hence, in this context, it should be considered that it would not be rigorously systematic to associate the observed phenomenon 
only with the number of layers. The development of a successful biosensing system taking advantages of such phenomenon would require a careful optimization of the mentioned parameters given the relevance in the corresponding photoluminescent effect.

\subsection{Lateral size}

Several factors related to the GO synthesis process lead to the ultimate lateral size, including original size of the precursor material (typically graphite), cross-planar oxidation of the precursor material, elastic strain energy, crack formation energy, interaction energy between layers and tensile stress buildup in the sheets. ${ }^{[18]}$ However, lateral size can also be respectively selected using specialized methods such as $\mathrm{KMnO}_{4}-\mathrm{H}_{2} \mathrm{SO}_{4}$ oxidation, ${ }^{[19]} \mathrm{pH}$-assisted selective sedimentation, ${ }^{[20]}$ controlled directional freezing, ${ }^{[21]}$ centrifugation ${ }^{[22]}$ and filtration through membranes among others. ${ }^{[23]}$

The literature reports a myriad of biosensing systems based on the high affinity between GO and single-stranded DNA, ${ }^{[24]}$ which can be labelled with a fluorophore that is quenched by the same interaction. In this context, the complex GO/fluorophore-conjugated single-strandedDNA can be easily utilized as a photoluminescent probe whose photoluminescent intensity dramatically increases upon target interaction due to the poor affinity between doublestranded DNA and GO. ${ }^{[1]}$ Zhang and colleagues reported that GO is complexed with DNA via a size dependent relationship and demonstrated that GO of $\sim 200 \mathrm{~nm}$ exhibited the highest photoluminescent quenching efficiency, whereas GO of $\sim 40 \mathrm{~nm}$ showed much lower quenching capability, see Figure 2A. Importantly, they discuss that "when the nanometer size of GO is decreased to $40 \mathrm{~nm}$, the charge density increases because of the repeated oxidation, and the negative charges provide a reverse force that drives the DNA apart from GO, which leads to lower quenching efficiency". Furthermore, they proved that the sensitivity and dynamic range of a biosensing system targeting metal ions can be modulated according to the lateral size of the employed GO, achieving the wider dynamic range using GO of $\sim 200 \mathrm{~nm}$ (1 
$-40 \mathrm{nM})$ and the lower limit of detection employing GO of $\sim 40 \mathrm{~nm}(0.1 \mathrm{nM}) .{ }^{[25]}$ Similarly, Prof. Dal-Hee Min group performed an exhaustive analysis of the interaction between DNA and GO with different sizes, that is to say, nanometer sized GO $(\sim 70 \mathrm{~nm})$ and micrometer sized GO sheets $(\sim 3 \mu \mathrm{m})$. This research team concluded that DNA showed faster adsorption kinetics utilizing nanometric $\mathrm{GO},{ }^{[26]}$ which can be a useful parameter to consider when designing biosensing platforms requiring a quick response.

Study of the interface between GO and bioreceptors is crucial in the design of biosensors. Tu et al. have widely discussed the interaction of graphene derivatives and biointerfaces, particularly pathogen, mammalian cells and tissue. ${ }^{[27]}$ Interaction of GO with cells is not only important in biosensing, but also in many other related applications such as bioimaging, therapy, drug delivery which are of great interest in achieving (together with biosensing/digansotics) and theranostic platforms/systems. ${ }^{[28]}$ Interestingly, biocompatibility and cellular uptake, which is pivotal in cell biosensors, have also been reported to be related to GO lateral size. Zhang et al. reported that cytotoxicity is size-dependent of GO lateral dimension. In fact, GO nanosheets $(\sim 34 \mathrm{~nm})$ provoked lower cytotoxicity towards HeLa cells than that of their sub-micrometric counterpart ( $\sim 147$ and $\sim 206 \mathrm{~nm}$, respectively), see Figure 2B. ${ }^{[19]}$ Likewise, antibacterial activity of GO (important to consider while designing pathogen biosensors) has been reported to be size-dependent, which may be able to compromise bacterial membrane integrity via oxidation stress, see Figure $2 C .{ }^{[28,29]}$ Furthermore, sharp edges, basal plane and mobility of GO flakes have been reported to modulate the antibacterial role of GO. ${ }^{[30]}$ Nevertheless, antibacterial activity of GO is still controversial since contradictory studies can be found and discussed in the literature. ${ }^{[28]}$ Cellular uptake of GO can also be enhanced by employing nanometric GO. Moreover, Zhang and co-workers discussed that the observed cytotoxicity can be attributed to membrane damage, where nanosheets are less threatening. Such evidence should be carefully considered 
while engineering cell-based sensors using GO. Furthermore, they also observed that HeLa cells underwent changes in their microenvironment due to uptake of GO, eventually causing a relative cytotoxicity. However, they highlight that nanometric GO proved the best biocompatibility. ${ }^{[19]}$ It is worth to spot that another study reported that surface charge also plays a critical role in cellular uptake. In fact, cellular uptake efficiency of positively charged thermally reduced GO sheets did not depend on the studied sizes $(\sim 200,550$ and $\sim 1000 \mathrm{~nm})$ and is carried out via phagocytosis and clathrin-mediated endocytosis pathways. In contrast, cellular uptake efficiency of the counterpart material with a negative surface charge is strongly modulated by lateral size. ${ }^{[31]}$

RNA possesses the capability to encode structural and functional information, facilitating potential strategies for therapy and diagnostics. ${ }^{[32]}$ In this context, GO could be utilized as a nanocarrier platform for small-interfering RNA (siRNA) delivery with interest also for future theranostic applications. However, siRNA has been reported to undergo structural alteration according to the GO lateral size interacting with this type of RNA. Actually, GO of $300 \pm 200$ nm was observed to intercalate between the double strands and trigger grave harm on the RNA conformation, whereas GO of $3 \pm 1 \mu \mathrm{m}$ reduces the A-helix pitch. In accordance to this, "GO can efficiently unwind, displace and partially denature the double siRNA strands, thus likely altering its biological activity". ${ }^{[33]}$ Hence, it is suggested to utilize functionalized GO in gene delivery or silencing approaches; for example, polyethyleneimine functionalization was discovered to hinder partial denaturation effect of GO upon interaction with siRNA. ${ }^{[33]}$ It is well known that photoluminescent emission wavelength of nanomaterials can be tuned according to their size. ${ }^{[34]}$ Zhang and colleagues studied how GO nanoflakes (graphene quantum dots) with average lateral sizes of 5.5, 12.5, and $16 \mathrm{~nm}$ exhibit different photoluminescent emission peaks; leading to blue, green and orange photoluminescence, respectively. ${ }^{[35]}$ Likewise, Mei et al., reported this size-dependent photoluminescent 
phenomenon. ${ }^{[36]}$ Apart from bioimaging approaches, this can be judiciously considered in multiplexed biosensing applications.

\subsection{Oxidation degree}

It is well known that GO possesses carboxyl functional groups (mainly at the lattice edges), ester, hydroxyl and epoxide moieties (on the basal plane of the lattice). GO oxidation degree can be modulated using several chemical routes based on reducing agents, which result in graphene derivatives with different properties. ${ }^{[37]}$ Alternatively, GO can be thermally reduced. ${ }^{[38]}$ The overall distribution of the aforementioned oxygen-containing groups is also crucial in the physicochemical properties of GO. Actually, carbon / oxygen $(\mathrm{C} / \mathrm{O})$ ratio of GO strongly modulates the photoluminescence quenching capabilities of GO and its binding interactions with single-stranded oligonucleotides. Hong and colleagues demonstrated how GO sub-micrometric flakes with similar sizes and thicknesses but with different $\mathrm{C} / \mathrm{O}$ rations behave differently in this context. They explored GO with $\mathrm{C} / \mathrm{O}$ ratios of 1.9, 1.6, 1.3 and 1.1, respectively, underscoring that the $\mathrm{GO}$ with higher $\mathrm{C} / \mathrm{O}$ is more efficient as both, a photoluminescence quenching agent and a single-stranded oligonucleotides adsorbing agent. ${ }^{[39]}$ Figure 3A depicts these phenomena. Moreover, it is worth mentioning that carboxyl groups in GO have been reported to provide a driving force to detach oligonucleotides from GO surface upon target interaction. ${ }^{[26]}$ These parameters are also important to consider in GObased biosensing, as they affect the overall analytical performance.

The demonstration of rationally designed control of GO photoluminescence via oxidation levels is quite challenging. More in-depth research is required since oxidation grade in addition to layer numbers and lateral size should be carefully and systematically controlled. Generally, GO photoluminescence is excited via UV light (around $365 \mathrm{~nm}$ ). According to the findings of Zhang et al., the photoluminescent emission of GO nanosheets was mainly associated with peripheral carboxylic groups. ${ }^{[35]}$ Moreover, bi/few-layers GO microsheets that 
underwent gradual deoxygenation, via thermal reduction, led to GO with different oxygenated functional groups corresponding to different photoluminescent peaks as follows; the yellowred emission of the studied GO (maximum peak at c.a. $610 \mathrm{~nm}$ ) was mainly associated with epoxy/hydroxyl groups, whereas the blue emission (maximum peak at c.a. $500 \mathrm{~nm}$ ) was mainly ascribed to the observed carbonyl functional groups. ${ }^{[40]}$ Jang and colleagues synthesized and explored several suspensions of GO nanosheets with differing oxidation degree and uniform lateral size $(3.37 \mathrm{~nm})$. They reported that the photoluminescent emission can be red-shifted from sky-blue (maximum peak at c.a. $443 \mathrm{~nm}$ ) to greenish-yellow (maximum peak at c.a. $528 \mathrm{~nm}$ ) by increasing the oxygen content in the explored material, see Figure 3B. Importantly the thermal conductivity of GO has also been reported to be strongly related to the abundance of oxygen-containing groups. Xin et al., demonstrated that at a coverage of $0.5 \%$ of oxygen-containing groups, the thermal conductivity of GO decreased around $50 \%$ compared to that of pristine graphene. ${ }^{[41]}$ Hence, the thermal conductivity of the employed GO should be seriously considered in biosensing systems based on refractive index changes as the refractive index of graphene derivatives can be affected due to temperature changes. ${ }^{[42,43]}$ Furhermore, this phenomenon can also be judiciously considered in thermalbased biosensing approaches.

As mentioned above, interaction of GO with cells is very important in bioanalytical applications. For example, such applications may allow for the achievement of new insights related to vital cellular tasks and cell-based therapy. ${ }^{[44,45]}$ Generally GO nanosheets are reported to be biocompatible. ${ }^{[46]}$ Moreover, cytotoxicity of sub-micrometric GO and reduced GO with uniform lateral size $(0.4-0.8 \mu \mathrm{m})$ has also been systematically studied by Das and colleagues. This research team concluded that GO was observed to be more toxic than its reduced counterpart. Importantly, they highlight that oxidative stress induced cytotoxicity can be hindered by decreasing oxygen functional group density on graphene derivatives as depicted in Figure 3C. ${ }^{[47]}$ Compatibility in vivo in tissue of GO microsheets with differing 
oxidation degrees has also been studied by Sydlik and co-workers using mice as animal model.

On the one hand, GO with higher oxidation degree led to increased accumulation of monocytes and an enhanced pro-inflammatory environment after intraperitoneal administration. On the other hand, reduced GO was observed to be more rapidly cleared from the intraperitoneal space and proven less chronic inflammation. However, the usage of GO microsheets with the same lateral size was not taken into consideration. ${ }^{[48]}$ This would have forcefully demonstrated the effect of GO oxidation grade in the corresponding biocompatibility study. It is worth mentioning that intact GO sub-micrometric sheets chemically functionalized with a stable radiometal chelating agent were found in the urine of injected mice (after intravenous administration). This suggests that chemical functionalization of graphene derivatives may enable control of the interaction of GO sheets with physiological interfaces ${ }^{[49]}$ and the outcomes of this phenomenon, ${ }^{[50]}$ all with interest not only for therapy but also biosensing/diagnostic applications.

\section{Innovative trends of GO in optical biosensing}

In our previous review article, ${ }^{[1]}$ we described a detailed overview on the fundamental understanding of chemical and physical features and mechanisms in GO-based optical biosensing. Due to the excellent photoluminescent quenching capabilities of GO and the aforementioned advantageous interaction between GO and single stranded oligonucleotidesbased probes followed by the low affinity between these probes and GO upon analyte recognition, ${ }^{[1]}$ the vast majority of GO-based optical biosensors rely on biosensing photoluminescent probes switched off/on by these highly specific interactions occurring in colloidal suspensions. Importantly, this bioanalytical strategy can be enhanced by designing probes with a significant difference in their adsorption energy via GO ${ }^{[51]}$ It is worth mentioning that this biosensing system has also been automated using microfluidics and simple optical analyzers. ${ }^{[52]}$ Moreover, this biosensing mechanism has been extended to 
aptamer selection, ${ }^{[53]}$ biomolecular logic gates operations, ${ }^{[54,55]}$ in situ live cell sensing, ${ }^{[56-58]}$ and drug monitoring. ${ }^{[59]}$ In addition GO has been proposed as an enzyme mimic agent that can be exploited in advantageous luminogenic and colorimetric assays, ${ }^{[60,61]}$ with interest as enzyme mimicking-based biosensing systems. Overall, these GO-based approaches are widely covered by recent review articles. ${ }^{[24,62-64]}$ Hence, we will focus this section on a critical overview of biosensing approaches involving the solid phase and label-free techniques by discussing representative examples from the functional point of view, that is, highlighting the critical role of GO.

\subsection{The solid phase opens up new opportunities in GO-based biosensing devices} The usage of GO as a biosensing platform in the solid phase has led to conceptually new biosensing devices. As far as we are concerned, Furukawa and colleagues introduced a GObased biosensing approach operating in the solid phase for the first time. Using coupling chemistry, they anchored fluorophore-labelled aptamers targeting a model protein (thrombin) on a single GO flake. ${ }^{[65]}$ Apart from a simple biosensing platform whose photoluminescence turns on upon analyte recognition, this enables a biosensing strategy amenable to multiplexed detection, miniaturization and integration via lab-on-a-chip devices, ${ }^{[66,67]}$ see Figure 4A. GOcoated surfaces decorated with fluorophore-labelled aptamers can also be employed to determine the presence of the analyte (e.g. pathogens) since the biosensing probes experiment a detaching mechanism upon analyte detection, which recovers the previously quenched photoluminescence. This biosensing strategy can be carried out and interrogated via a microfluidic reaction chamber. ${ }^{[68]}$ In addition, biosensing photoluminescent inks based on GO have been formulated and printed on paper using a conventional ink-jet printer. ${ }^{[69]}$ The formulation consist of aqueous suspensions of photoluminescent GO loaded with biorecognition probes-decorated silver nanoparticles (AgNPs). As AgNPs efficiently quench photoluminescent GO and such biosensing probes-decorated AgNPs are specifically detached 
from GO upon analyte detection, this phenomenon offers a straightforward off/on nanosensing mechanism. In addition, this approach can be extended to DNA, peptide and protein detection according to the utilized biorecognition probe, see Figure 4B. Moreover, photoluminescent GO nanosheets spotted on paper strips have been reported to be quenched upon phenols and polyphenols interaction, which has been interrogated using a smart-phone device as analytical tool for environmental and food samples monitoring. ${ }^{[0]}$ It is well-known that non-radiative energy transfer (NRET) can be strongly modulated by the distance between the involved donor and acceptor. Using graphene derivatives, this phenomenon is observable up to c.a. $30 \mathrm{~nm} \cdot{ }^{[14,71]}$ In this regard, innovative immunosensing have been demonstrated by using a) GO as acceptor, b) antibody-decorated quantum dots (Ab-QDs) as donors, and c) the analyte as a spacer between donor and acceptor. Hence, strong NRET is expected to occur only in the absence of the analyte, facilitating immunosensing platforms whose photoluminescent emission is dramatically triggered by analyte intercalation between GO and QDs. This biosensing mechanism employs a single type of antibody and has been successfully employed for pathogen detection via several formats based on the solid phase; including microarray technology, ${ }^{[72]}$ lateral flow strips (see Figure 4C), ${ }^{[73]}$ and paper-based devices. ${ }^{[74,75]}$ Although pathogens are a giant spacer (c.a. $\left.1 \mu \mathrm{m}\right)$ to avoid the aforementioned NRET, engineering new configurations, this biosensing concept has also been extended to protein detection in lateral flow strips, ${ }^{[76]}$ and GO-coated nanopaper substrates, see Figure 4D. ${ }^{[75]}$ Overall, these biosensing platforms were proven advantageous in terms of sensitivity and simplicity when compared with conventional immunoassays, as they are able to save time and precious bio-reagents (such as antibodies).

\subsection{GO in label-free optical biosensing}

Label-free optical biosensing offers several advantages, including high sensitivity, fast and real time analysis. Label-free biosensing can be based on highly sensitive modulations in the refractive index occurring at the sensing surface, which are provoked by biomolecular 
recognition events. However, as mentioned above, this type of biosensors might be affected by non-specific refractive index changes in the employed GO, which can be triggered by temperature changes. ${ }^{[77]}$ Consequently, temperature and GO oxidation degree should be carefully controlled in these approaches. Surface plasmon resonance (SPR) biosensors and optical waveguide biosensors rely on the aforementioned refractive index-dependent biosensing mechanism. ${ }^{[77-79]}$ Although label-free optical biosensing platforms have been reported to be enhanced by using structural properties of GO, as far as we are concerned, the elucidation of the optical/photonic properties or phenomena underlying such improvements have been scarcely elaborated until now, which can be subject of in-depth investigation. Generally, given its high surface area and oxygenated honeycomb lattice structure, GO is reported to facilitate the improvement of the density of biorecognition elements onto the sensing surface of label-free biosensing devices. ${ }^{[80]}$

Optical fibers are a particular example of optical waveguides. ${ }^{[81]}$ Chen and co-workers explored an optical fiber (particularly, dual-peak long period grating) decorated with GO and antibodies targeting anti-IgG, see Figure 5A. The authors reported that the studied biodetection platform surpasses its counterpart without GO due to the improved chemical bonding and physical adsorption of the biorecognition enabled by GO. ${ }^{[82]}$ In fact, the GOcoated fiber approach showed a limit of detection 10-fold lower than that of the non-coated fiber. Similarly, Kamil and colleagues described a GO-coated tapered optical fiber biosensor for Dengue detection. ${ }^{[83]}$ Decoration of other kind of optical waveguides with GO has been scarcely reported. However, this might lead to advantageous biosensing platforms exploiting light flow modulation or light polarization modulation as a transduction system. ${ }^{\left[{ }^{84-86]}\right.}$ Recently, Chung et al., reported the SPR enhancement at pristine graphene/metal ( $\mathrm{Au})$ interface. The authors highlighted that the enhanced refractive index sensitivity relies on induced surface dipole due to the charge transfer between the metallic film and pristine graphene. ${ }^{[87]}$ Moreover, spectroscopic ellipsometry revealed that GO-coated SPR substrates 
displayed much lower optical absorption in the visible range than its counterpart coated with pristine graphene. ${ }^{[88]}$ In fact, GO-coated SPR substrates have been reported to outperform the bioanalytical sensitivity of commercially available biochips containing a 3D linker. A 2.9-fold enhancement was discussed in this biosensing approach studied by Stebunov et al., ${ }^{[88]}$ see Figure 5B. Although the plasmonic nature of graphene derivatives mainly operates in the terahertz region, ${ }^{[89]}$ Primo and collaborators emphasized that the contribution of GO increased the explored electromagnetic field using visible light, thus playing a critical role in SPR enhanced by GO within the visible region. This research team engineered a SPR substrate based on a GO-coated Au surface for the screening of a cardiac biomarker (galectin-3), reaching a clinically relevant concentration range. ${ }^{[90]}$ Interestingly, Xiong and colleagues discussed that the thickness of GO-coated gold surfaces with a GO layer thickness of around hundreds of nanometers led to a significant improvement in SPR sensitivity given a modulation in the refractive index sensitivity caused by the studied GO coating. They also concluded that the SPR sensitivity was observed to be proportional to the thickness of the explored GO coating. ${ }^{[91]}$

It is well-known that Raman spectroscopy facilitates the determination of bio/molecules via their highly specific Raman scattering ${ }^{[92]}$ In this regard, surface enhanced Raman spectroscopy (SERS) can also be exploited as a powerful label-free bioanalytical platform. ${ }^{[93,94]}$ Generally, SERS relies on a sharp rise in the Raman scattering by means of the local amplification of the electromagnetic field experimented around noble metal nanomaterials, which is provoked by the excitation of localized surface plasmon resonances. ${ }^{[95]}$ In addition, composite materials based on noble metal nanoparticles and graphene derivatives have been reported to provide advantageous SERS substrates, see Figure 5C. ${ }^{[64,96]}$ Particularly, graphene derivatives-based SERS substrates enable the following advantages: i) A fluorescence-quenching nature offering the capability to analyze fluorescent analytes, even using an excitation laser close to the excitation wavelength of the analyte. ${ }^{[97]}$ ii) 
A surface passivation effect and chemical stability improvement, which protect noble metal surfaces from degradation or undesired interactions with the environment. ${ }^{[98,99]}$ iii) The ability to anchor organic compounds utilizing $\pi-\pi$ stacking interactions for highly sensitive analysis. ${ }^{[100-102]}$ iv) A charge transferring phenomenon that improves SERS performance. ${ }^{[103]}$ Interestingly, the 2D character of GO facilitates engineering of advantageous 3D SERS substrates with different numbers of $\mathrm{AgNp}$ layers intercalated, showing from picomolar to femtomolar sensitivity. ${ }^{[104]}$ Moreover, GO leads to generation of flexible membrane-like SERS substrates than can be utilized as pre-concentration platforms for highly sensitive analysis of large volumes. ${ }^{[105,106]} \mathrm{GO}$ has also been reported to endow SERS substrates with ultra-stable analytical behavior, thus enabling the analysis of the same Raman signature throughout tens of days. ${ }^{[106]}$ Notably, the literature describes particular SERS applications of compositesincorporating GO in several fields, ${ }^{[24,64]}$ including bio-imaging, ${ }^{[106,107]}$ biomarkers detection, ${ }^{[108-}$ ${ }^{110]}$ bacteria determination, ${ }^{[110]}$ pesticides monitoring, ${ }^{[111]}$ and food analysis among others. ${ }^{[112]}$

\section{Conclusion and future perspectives}

The exquisite physicochemical properties of GO are leading to advantageous biosensing approaches improving analytical sensitivity and analysis time. Apart from this, GO-based biosensing approaches can obviate the need for cumbersome conventional procedures and empower the capability to produce cost-effective devices, for example by simplifying manufacture processes and saving expensive bioreagents. In this context, GO-based optical biosensing approaches are already offering integrated smart devices. ${ }^{[113]}$ Nevertheless, there is a paucity of evaluation of GO-based biosensors in pre-clinical settings. Given the relevance of lateral size, number of layers and oxidation degree of GO in optical biosensing behavior, researchers and technologists working in this field are expected to be committed to characterize and describe these features in their approaches aiming at ensuring coherence and reproducibility following nanometrology as well. Graphene derivatives are known to interact 
virtually with the whole spectrum, that is, from UV rays to terahertz radiation. ${ }^{[114]}$ In fact, exciting efforts focused on near-infrared light-activated therapy and near-infrared light-based drug delivery are reported in the literature. ${ }^{[115]}$ However, in spite of the strong relationship between therapy and diagnostics, biosensing applications of GO based on infrared rays or terahertz waves have been scarcely explored yet. Moreover, the significance of lateral size, number of layers and oxidation degree of GO is still little explored in terahertz radiation, infrared wavelengths and label-free biosensing. In addition, heterostructures based on graphene derivatives and other 2D materials such as molybdenum disulfide, hexagonal boron nitride and black phosphorus are expected to bring breakthroughs in biosensing as they enhance the light-matter interaction. ${ }^{[114]}$ Consequently, these emerging fields are full of challenges and opportunities in optical biosensing.

\section{Acknowledgements}

E. M.-N. acknowledges the financial support from CONACYT (Mexico, Grant 293523) and National System of Researchers, CONACYT (Mexico, Grant 74314). A.M. thanks the support from European Commission through the Graphene Flagship Core 2 project. ICN2 acknowledges the support of the Spanish MINECO through the Severo Ochoa Centers of Excellence Program under Grant SEV2201320295. ICN2 is also funded by the CERCA Program/Generalitat de Catalunya.

Received: ((will be filled in by the editorial staff))

Revised: ((will be filled in by the editorial staff)) Published online: ((will be filled in by the editorial staff))

\section{References}

[1] E. Morales-Narváez, A. Merkoçi, Adv. Mater. 2012, 24, 3298.

[2] A. Bianco, H.-M. Cheng, T. Enoki, Y. Gogotsi, R. H. Hurt, N. Koratkar, T. Kyotani, M. Monthioux, C. R. Park, J. M. D. Tascon, J. Zhang, Carbon 2013, 65, 1.

[3] P. Wick, A. E. Louw-Gaume, M. Kucki, H. F. Krug, K. Kostarelos, B. Fadeel, K. A. Dawson, A. Salvati, E. Vázquez, L. Ballerini, M. Tretiach, F. Benfenati, E. Flahaut, L. Gauthier, M. Prato, A. Bianco, Angew. Chem. Int. Ed. 2014, 53, 7714.

[4] M. Goumri, B. Lucas, B. Ratier, M. Baitoul, Opt. Mater. 2016, 60, 105.

[5] E. Morales-Narváez, L. F. Sgobbi, S. A. S. Machado, A. Merkoçi, Prog. Mater. Sci. 2017, 86, 1 .

[6] L. Baptista-Pires, C. C. Mayorga-Martínez, M. Medina-Sánchez, H. Montón, A. Merkoçi, ACS Nano 2016, 10, 853. 
[7] D. Joung, T. Gu, J.-H. Cho, ACS Nano 2016, 10, 9586.

[8] Y.-K. Kim, D.-H. Min, Langmuir 2014, 30, 12675.

[9] L. Tong, W. Qi, M. Wang, R. Huang, R. Su, Z. He, Small 2016, 12, 3433.

[10] S.-H. Hong, J.-K. Song, Small 2017, 13, 1603125.

[11] L. Tong, W. Qi, M. Wang, R. Huang, R. Su, Z. He, Small 2017, 13, 1700102.

[12] C. H. Lee, J. Yu, Y. Wang, A. Y. L. Tang, C. W. Kan, J. H. Xin, $R S C A d v$. 2018, 8, 16593.

[13] M. Qin, M. Sun, R. Bai, Y. Mao, X. Qian, D. Sikka, Y. Zhao, H. J. Qi, Z. Suo, X. He, Adv. Mater. 2018, 30, 1800468.

[14] E. Morales-Narváez, L. Baptista-Pires, A. Zamora-Gálvez, A. Merkoçi, Adv. Mater. 2017, 29, 1604905.

[15] Z. Chen, S. Berciaud, C. Nuckolls, T. F. Heinz, L. E. Brus, ACS Nano 2010, 4, 2964.

[16] X. T. Guo, Z. Hua Ni, C. Yan Liao, H. Yan Nan, Y. Zhang, W. Wei Zhao, W. Hui Wang, Appl. Phys. Lett. 2013, 103, 201909.

[17] Y. Dong, C. Chen, X. Zheng, L. Gao, Z. Cui, H. Yang, C. Guo, Y. Chi, C. M. Li, J. Mater. Chem. 2012, 22, 8764.

[18] S. Pan, I. A. Aksay, ACS Nano 2011, 5, 4073.

[19] H. Zhang, C. Peng, J. Yang, M. Lv, R. Liu, D. He, C. Fan, Q. Huang, ACS Appl. Mater. Interfaces 2013, 5, 1761.

[20] X. Wang, H. Bai, G. Shi, J. Am. Chem. Soc. 2011, 133, 6338.

[21] H. Geng, B. Yao, J. Zhou, K. Liu, G. Bai, W. Li, Y. Song, G. Shi, M. Doi, J. Wang, J. Am. Chem. Soc. 2017, 139, 12517.

[22] A. M. Gravagnuolo, E. Morales-Narváez, S. Longobardi, E. T. da Silva, P. Giardina, A. Merkoçi, Adv. Funct. Mater. 2015, 25, 2771.

[23] Chen Ji, Li Yingru, Huang Liang, Jia Naer, Li Chun, Shi Gaoquan, Adv. Mater. 2015, $27,3654$.

[24] J. Kim, S.-J. Park, D.-H. Min, Anal. Chem. 2017, 89, 232.

[25] H. Zhang, S. Jia, M. Lv, J. Shi, X. Zuo, S. Su, L. Wang, W. Huang, C. Fan, Q. Huang, Anal. Chem. 2014, 86, 4047.

[26] J. Lee, Y. Yim, S. Kim, M.-H. Choi, B.-S. Choi, Y. Lee, D.-H. Min, Carbon 2016, 97, 92.

[27] Tu Zhaoxu, Guday Guy, Adeli Mohsen, Haag Rainer, Adv. Mater. 2018, o, 1706709.

[28] Bitounis Dimitrios, Ali-Boucetta Hanene, Hong Byung Hee, Min Dal-Hee, Kostarelos Kostas, Adv. Mater. 2013, 25, 2258.

[29] S. Liu, M. Hu, T. H. Zeng, R. Wu, R. Jiang, J. Wei, L. Wang, J. Kong, Y. Chen, Langmuir 2012, 28, 12364.

[30] K. H. Tan, S. Sattari, I. S. Donskyi, J. L. Cuellar-Camacho, C. Cheng, K. Schwibbert, A. Lippitz, W. E. S. Unger, A. Gorbushina, M. Adeli, R. Haag, Nanoscale 2018, 10, 9525.

[31] Tu Zhaoxu, Achazi Katharina, Schulz Andrea, Mülhaupt Rolf, Thierbach Steffen, Rühl Eckart, Adeli Mohsen, Haag Rainer, Adv. Funct. Mater. 2017, 27, 1701837.

[32] S. H. Lee, B. H. Chung, T. G. Park, Y. S. Nam, H. Mok, Acc. Chem. Res. 2012, 45, 1014.

[33] G. Reina, N. D. Q. Chau, Y. Nishina, A. Bianco, Nanoscale 2018, 10, 5965.

[34] X. Hai, J. Feng, X. Chen, J. Wang, J. Mater. Chem. B 2018, 6, 3219.

[35] F. Zhang, F. Liu, C. Wang, X. Xin, J. Liu, S. Guo, J. Zhang, ACS Appl. Mater. Interfaces 2016, 8, 2104.

[36] Q. Mei, J. Chen, J. Zhao, L. Yang, B. Liu, R. Liu, Z. Zhang, ACS Appl. Mater. Interfaces 2016, 8, 7390.

[37] C. K. Chua, M. Pumera, Chem. Soc. Rev. 2014, 43, 291. 
[38] P. V. Kumar, N. M. Bardhan, G.-Y. Chen, Z. Li, A. M. Belcher, J. C. Grossman, Carbon 2016, 100, 90.

[39] B. J. Hong, Z. An, O. C. Compton, S. T. Nguyen, Small 2012, 8, 2469.

[40] R. M. Ray, A. Midya, C. Narayana, S. K, Nanotechnology 2014, 25, 495704.

[41] X. Mu, X. Wu, T. Zhang, D. B. Go, T. Luo, Sci. Rep. 2014, 4, 3909.

[42] Jun Zhang and Guozhen Liao and Shaoshen Jin and Dong Cao and Qingsong Wei and Huihui Lu and Jianhui Yu and Xiang Cai and Shaozao Tan and Yi Xiao and Jieyuan Tang and Yunhan Luo and Zhe Chen, Laser Phys. Lett. 2014, 11, 035901.

[43] L. Li, Z. Feng, X. Qiao, H. Yang, R. Wang, D. Su, Y. Wang, W. Bao, J. Li, Z. Shao, M. Hu, IEEE Sens. J. 2015, 15, 505.

[44] T.-H. Kim, D. Lee, J.-W. Choi, Biosens. Bioelectron. 2017, 94, 485.

[45] G. M. Vlăsceanu, R.-M. Amărandi, M. Ioniță, T. Tite, H. Iovu, L. Pilan, J. S. Burns, Biosens. Bioelectron. 2018, 117, 283.

[46] Haque Enamul, Kim Jeonghun, Malgras Victor, Reddy Kakarla Raghava, Ward Alister C., You Jungmok, Bando Yoshio, Hossain Md. Shahriar A., Yamauchi Yusuke, Small Methods 2018, 0, 1800050.

[47] S. Das, S. Singh, V. Singh, D. Joung, J. M. Dowding, D. Reid, J. Anderson, L. Zhai, S. I. Khondaker, W. T. Self, S. Seal, Part. Part. Syst. Charact. 2013, 30, 148.

[48] S. A. Sydlik, S. Jhunjhunwala, M. J. Webber, D. G. Anderson, R. Langer, ACS Nano 2015, 9, 3866.

[49] D. A. Jasim, C. Ménard-Moyon, D. Bégin, A. Bianco, K. Kostarelos, Chem. Sci. 2015, $6,3952$.

[50] N. Bernabò, A. Fontana, M. R. Sanchez, L. Valbonetti, G. Capacchietti, R. Zappacosta, L. Greco, M. Marchisio, P. Lanuti, E. Ercolino, B. Barboni, Carbon 2018, 129, 428.

[51] B. Liu, Z. Sun, X. Zhang, J. Liu, Anal. Chem. 2013, 85, 7987.

[52] X. Weng, S. Neethirajan, Biosens. Bioelectron. 2016, 85, 649.

[53] J.-W. Park, R. Tatavarty, D. W. Kim, H.-T. Jung, M. B. Gu, Chem. Commun. 2012, 48, 2071.

[54] L. Wang, J. Zhu, L. Han, L. Jin, C. Zhu, E. Wang, S. Dong, ACS Nano 2012, 6, 6659.

[55] C. Zhou, D. Liu, C. Wu, Y. Liu, E. Wang, Nanoscale 2016, 8, 17524.

[56] S.-R. Ryoo, J. Lee, J. Yeo, H.-K. Na, Y.-K. Kim, H. Jang, J. H. Lee, S. W. Han, Y. Lee, V. N. Kim, D.-H. Min, ACS Nano 2013, 7, 5882.

[57] Y. Wang, Z. Li, T. J. Weber, D. Hu, C.-T. Lin, J. Li, Y. Lin, Anal. Chem. 2013, 85, 6775.

[58] B. Esteban-Fernández de Ávila, A. Martín, F. Soto, M. A. Lopez-Ramirez, S. Campuzano, G. M. Vásquez-Machado, W. Gao, L. Zhang, J. Wang, ACS Nano 2015, 9, 6756.

[59] H. Jang, S.-R. Ryoo, M. J. Lee, S. W. Han, D.-H. Min, Mol. Cells 2013, 35, 269.

[60] Y. Song, K. Qu, C. Zhao, J. Ren, X. Qu, Adv. Mater. 2010, 22, 2206.

[61] S. Wang, R. Cazelles, W.-C. Liao, M. Vázquez-González, A. Zoabi, R. Abu-Reziq, I. Willner, Nano Lett. 2017, 17, 2043.

[62] B. Chen, Q. Su, W. Kong, Y. Wang, P. Shi, F. Wang, J. Mater. Chem. B 2018, 6, 2924.

[63] L. Tang, Y. Wang, J. Li, Chem. Soc. Rev. 2015, 44, 6954.

[64] J. Lee, J. Kim, S. Kim, D.-H. Min, Graphene-Based Mater. Nanomedicine 2016, 105, 275.

[65] K. Furukawa, Y. Ueno, E. Tamechika, H. Hibino, J. Mater. Chem. B 2013, 1, 1119.

[66] Y. Ueno, K. Furukawa, K. Matsuo, S. Inoue, K. Hayashi, H. Hibino, Y. Sato, 2013, 17th International Conference on Miniaturized Systems for Chemistry and Life Sciences, MicroTAS 2013, pp. 814-816.

[67] Y. Ueno, K. Furukawa, K. Matsuo, S. Inoue, K. Hayashi, H. Hibino, Anal. Chim. Acta 2015, 866, 1. 
[68] P. Zuo, X. Li, D. C. Dominguez, B.-C. Ye, Lab. Chip 2013, 13, 3921.

[69] Mei Qingsong, Zhang Zhongping, Angew. Chem. Int. Ed. 2012, 51, 5602.

[70] R. Álvarez-Diduk, J. Orozco, A. Merkoçi, Sci. Rep. 2017, 7, 976.

[71] L. Gaudreau, K. J. Tielrooij, G. E. D. K. Prawiroatmodjo, J. Osmond, F. J. G. de Abajo, F. H. L. Koppens, Nano Lett. 2013, 13, 2030.

[72] E. Morales-Narváez, A.-R. Hassan, A. Merkoçi, Angew. Chem. Int. Ed. 2013, 52, 13779.

[73] E. Morales-Narváez, T. Naghdi, E. Zor, A. Merkoçi, Anal. Chem. 2015, 87, 8573.

[74] E. Morales-Narváez, H. Golmohammadi, T. Naghdi, H. Yousefi, U. Kostiv, D. Horák, N. Pourreza, A. Merkoçi, ACS Nano 2015, 9, 7296.

[75] N. Cheeveewattanagul, E. Morales-Narváez, A.-R. H. A. Hassan, J. F. Bergua, W. Surareungchai, M. Somasundrum, A. Merkoçi, Adv. Funct. Mater. 2017, 27, 1702741.

[76] A. Zamora-Gálvez, E. Morales-Narváez, J. Romero, A. Merkoçi, Biosens. Bioelectron. 2018, 100, 208.

[77] Y. Zhao, X. Li, X. Zhou, Y. Zhang, Sens. Actuators B Chem. 2016, 231, 324.

[78] S. Zeng, D. Baillargeat, H.-P. Ho, K.-T. Yong, Chem. Soc. Rev. 2014, 43, 3426.

[79] P. Kozma, F. Kehl, E. Ehrentreich-Förster, C. Stamm, F. F. Bier, Biosens. Bioelectron. 2014, 58, 287.

[80] Zhang Hua, Sun Ying, Gao Shang, Zhang Jia, Zhang Hanqi, Song Daqian, Small 2013, 9, 2537.

[81] Blue Robert, Uttamchandani Deepak, J. Biophotonics 2015, 9, 13.

[82] C. Liu, Q. Cai, B. Xu, W. Zhu, L. Zhang, J. Zhao, X. Chen, Biosens. Bioelectron. 2017, 94, 200.

[83] Y. Mustapha Kamil, M. H. Abu Bakar, M. H. Yaacob, A. Syahir, H. N. Lim, M. A. Mahdi, IEEE J. Sel. Top. Quantum Electron. 2019, 25, 1.

[84] W. H. Lim, Y. K. Yap, W. Y. Chong, C. H. Pua, N. M. Huang, R. M. De La Rue, H. Ahmad, Opt. Express 2014, 22, 11090.

[85] Harith Ahmad and Soroush Soltani and Mohammad Faizal Ismail and Kavintheran Thambiratnam and Chong Wu Yi and Moh Yasin, Laser Phys. 2018, 28, 076001.

[86] W. Y. Chong, W. H. Lim, Y. K. Yap, C. K. Lai, R. M. De La Rue, H. Ahmad, Sci. Rep. 2016, 6, 23813.

[87] Chung Kyungwha, Lee June Sang, Kim Eunah, Lee Kyung-Eun, Kim Kiheung, Lee Junghyun, Kim Donghyun, Kim Sang Ouk, Jeon Seokwoo, Park Hyesung, Kim DongWook, Kim Dong Ha, Adv. Mater. Interfaces 2018, 0, 1800433.

[88] Y. V. Stebunov, O. A. Aftenieva, A. V. Arsenin, V. S. Volkov, ACS Appl. Mater. Interfaces 2015, 7, 21727.

[89] A. N. Grigorenko, M. Polini, K. S. Novoselov, Nat Photon 2012, 6, 749.

[90] E. N. Primo, M. J. Kogan, H. E. Verdejo, S. Bollo, M. D. Rubianes, G. A. Rivas, ACS Appl. Mater. Interfaces 2018, 10, 23501.

[91] Xin Xiong, Yaofei Chen, Hao Wang, Shiqi Hu, Yunhan Luo, Jiangli Dong, Wenguo Zhu, Wentao Qiu, Heyuan Guan, Huihui Lu, Jianhui Yu, Jun Zhang, Zhe Chen, ACS Appl. Mater. Interfaces 2018, DOI 10.1021/acsami.8b11424.

[92] C. L. Haynes, A. D. McFarland, R. P. V. Duyne, Anal. Chem. 2005, 77, 338 A.

[93] D. Cialla-May, X.-S. Zheng, K. Weber, J. Popp, Chem. Soc. Rev. 2017, 46, 3945.

[94] C. Zong, M. Xu, L.-J. Xu, T. Wei, X. Ma, X.-S. Zheng, R. Hu, B. Ren, Chem. Rev. 2018, $118,4946$.

[95] M. Gómez, M. Lazzari, Mater. Today 2014, 17, 358.

[96] W. Xu, N. Mao, J. Zhang, Small 2013, 9, 1206.

[97] R. Goul, S. Das, Q. Liu, M. Xin, R. Lu, R. Hui, J. Z. Wu, Carbon 2017, 111, 386.

[98] J. C. Reed, H. Zhu, A. Y. Zhu, C. Li, E. Cubukcu, Nano Lett. 2012, 12, 4090.

[99] S. Suzuki, M. Yoshimura, Sci. Rep. 2017, 7, 14851. 
[100] X. Li, W. C. H. Choy, X. Ren, D. Zhang, H. Lu, Adv. Funct. Mater. 2014, 24, 3114.

[101] W. Ren, M. Qin, X. Hu, F. Li, Y. Wang, Y. Huang, M. Su, W. Li, X. Qian, K. Tang, Y. Song, Anal. Chem. 2018, 90, 6371.

[102] A. M. Gravagnuolo, E. Morales-Narváez, C. R. S. Matos, S. Longobardi, P. Giardina, A. Merkoçi, Adv. Funct. Mater. 2015, 25, 6084.

[103] Y.-K. Kim, G. Ok, S.-W. Choi, H. Jang, D.-H. Min, Nanoscale 2017, 9, 5872.

[104] S. R. Das, Q. Nian, A. A. Cargill, J. A. Hondred, S. Ding, M. Saei, G. J. Cheng, J. C. Claussen, Nanoscale 2016, 8, 15870.

[105] L. Qu, N. Wang, H. Xu, W. Wang, Y. Liu, L. Kuo, T. P. Yadav, J. Wu, J. Joyner, Y. Song, H. Li, J. Lou, R. Vajtai, P. M. Ajayan, Adv. Funct. Mater. 2017, 27, 1701714.

[106] E. Rodríguez-Sevilla, G. V. Vázquez, E. Morales-Narváez, Adv. Opt. Mater. 2018, o, 1800548 .

[107] Q. Liu, L. Wei, J. Wang, F. Peng, D. Luo, R. Cui, Y. Niu, X. Qin, Y. Liu, H. Sun, J. Yang, Y. Li, Nanoscale 2012, 4, 7084.

[108] W. Ren, Y. Fang, E. Wang, ACS Nano 2011, 5, 6425.

[109] T. Demeritte, B. P. Viraka Nellore, R. Kanchanapally, S. S. Sinha, A. Pramanik, S. R. Chavva, P. C. Ray, ACS Appl. Mater. Interfaces 2015, 7, 13693.

[110] Z. Fan, R. Kanchanapally, P. C. Ray, J. Phys. Chem. Lett. 2013, 4, 3813.

[111] Z. Liu, Y. Wang, R. Deng, L. Yang, S. Yu, S. Xu, W. Xu, ACS Appl. Mater. Interfaces 2016, 8, 14160.

[112] Y. Xie, Y. Li, L. Niu, H. Wang, H. Qian, W. Yao, Talanta 2012, 100, 32.

[113] D. Quesada-González, A. Merkoçi, Chem. Soc. Rev. 2018, 47, 4697.

[114] F. Xia, H. Wang, D. Xiao, M. Dubey, A. Ramasubramaniam, Nat. Photonics 2014, 8, 899.

[115] V. Shanmugam, S. Selvakumar, C.-S. Yeh, Chem. Soc. Rev. 2014, 43, 6254.

[116] M.-H. Jang, H. D. Ha, E.-S. Lee, F. Liu, Y.-H. Kim, T. S. Seo, Y.-H. Cho, Small 2015, 11,3773 . 

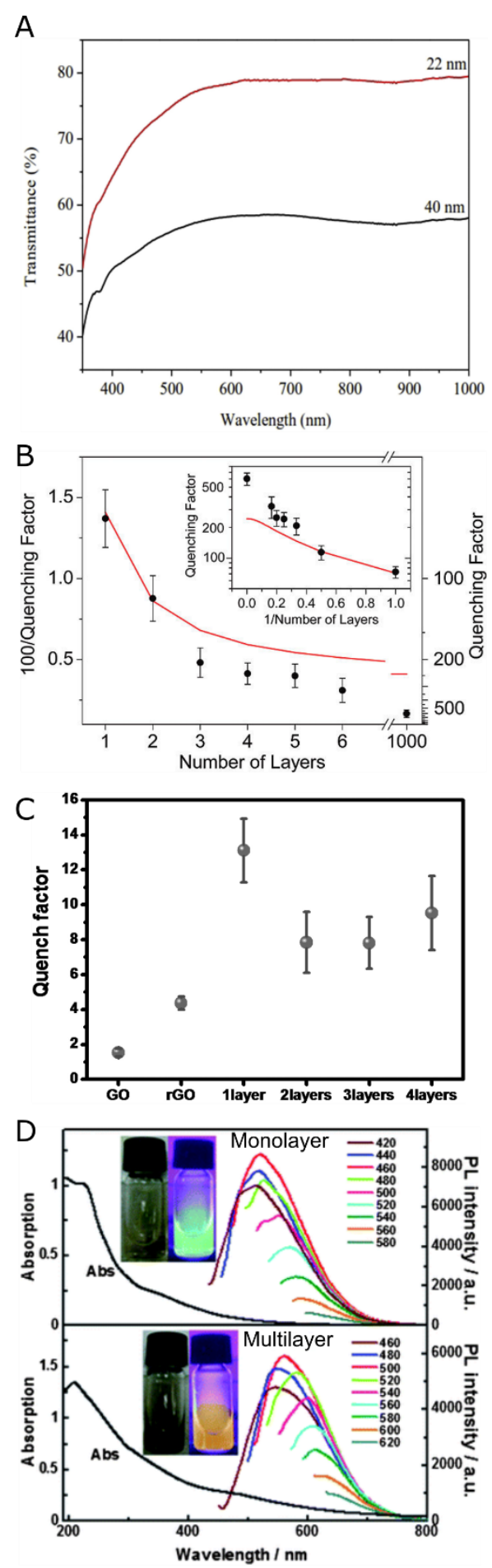

Figure 1. Layer number-related optical properties of GO. A. Transmittance and GO thickness. Adapted with permission from ${ }^{[4]}$. Copyright 2016 Elsevier. B-C. Controversial approaches on photoluminescence quenching factor using GO as quencher. B. Photoluminescence quenching efficiency increases with layer number. Adapted with permission from ${ }^{[15]}$. Copyright 2010 American Chemical Society. C. Photoluminescence quenching efficiency decreases with layer number. Adapted with permission from ${ }^{[16]}$. Copyright 2013 AIP Publishing. D.

Photoluminecence of monolayer and multilayer GO nanosheets. Adapted with permission from ${ }^{[17]}$. Copyright 2012 Royal Society of Chemistry. 

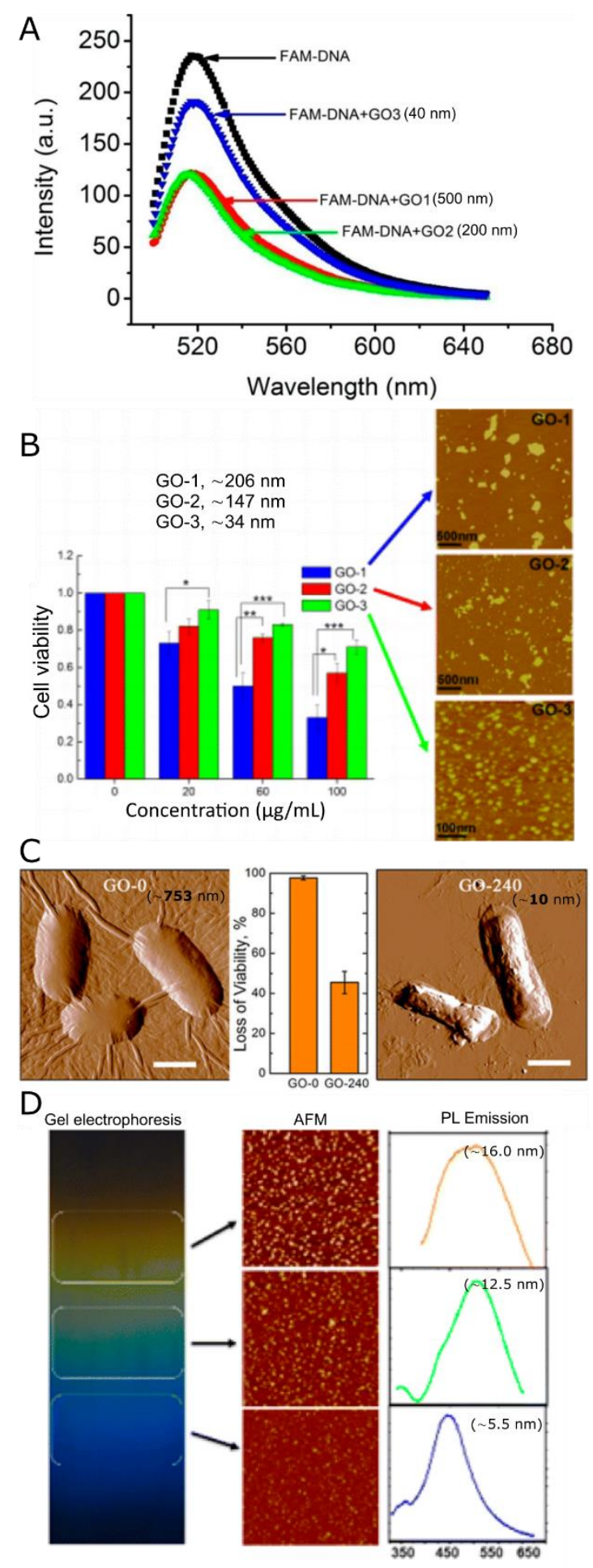

Figure 2. Properties and interactions of GO related to lateral size. A. GO lateral size and photoluminescence quenching ability. Adapted with permission from ${ }^{[25]}$. Copyright 2014 American Chemical Society. B. GO lateral size and cell viability. Adapted with permission from ${ }^{[19]}$. Copyright 2013 American Chemical Society. C. GO lateral size and Antibacterial activity. Adapted with permission from ${ }^{[29]}$. Copyright 2012 American Chemical Society. D. GO nanosheets and their photoluminescence. Adapted with permission from ${ }^{[35]}$. Copyright 2016 American Chemical Society. 

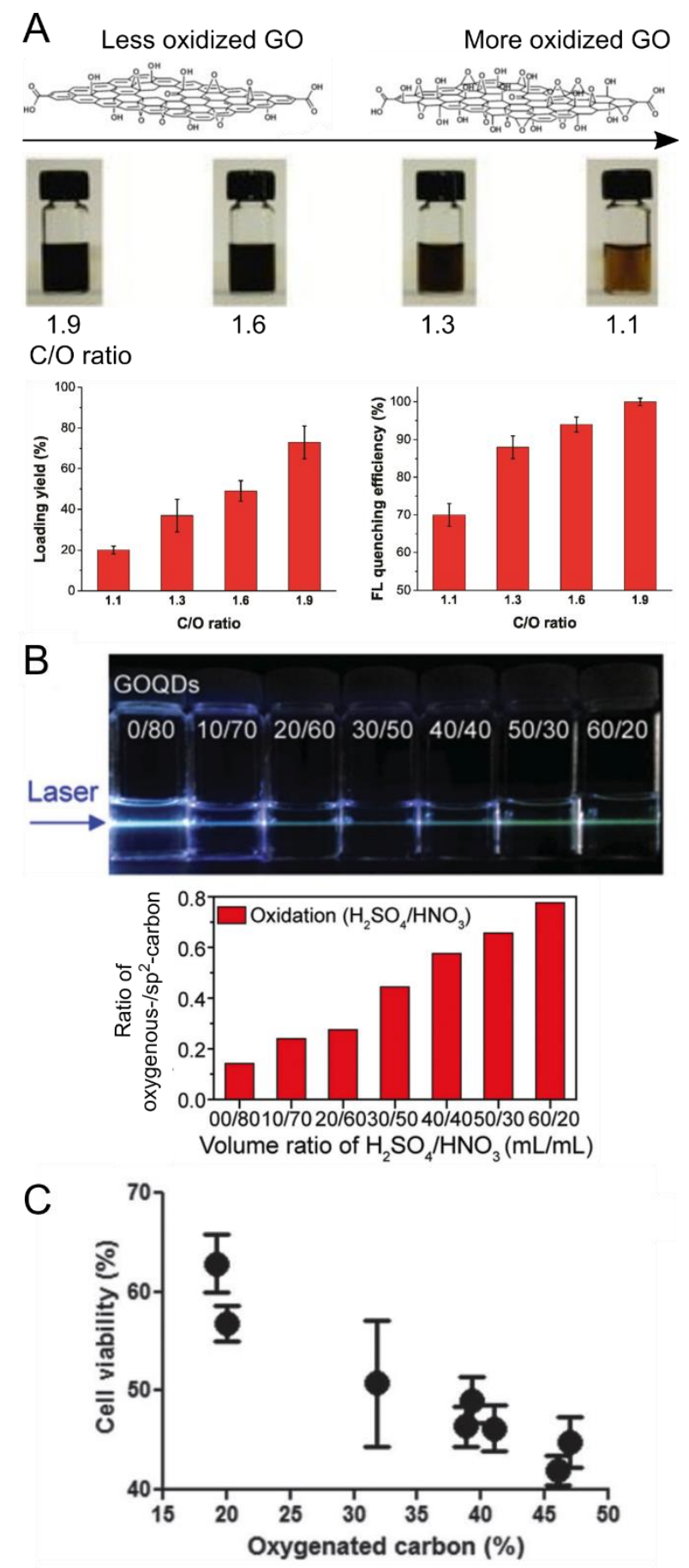

Figure 3. Properties and interactions of GO related to oxidation degree. A. Oxidation degree affects loading yield of single-stranded oligonucleotides and photoluminescence quenching efficiency. Adapted with permission from ${ }^{[39]}$. Copyright 2012 John Wiley and Sons. B.

Photoluminescent emission of GO nanosheets modulated by modifying the oxidation degree. Adapted with permission from ${ }^{[116]}$. Copyright 2015 John Wiley and Sons. C. Cell viability improves by decreasing the oxygen content in GO sub-micrometric sheets. Adapted with permission from ${ }^{[47]}$. Copyright 2013 John Wiley and Sons. 

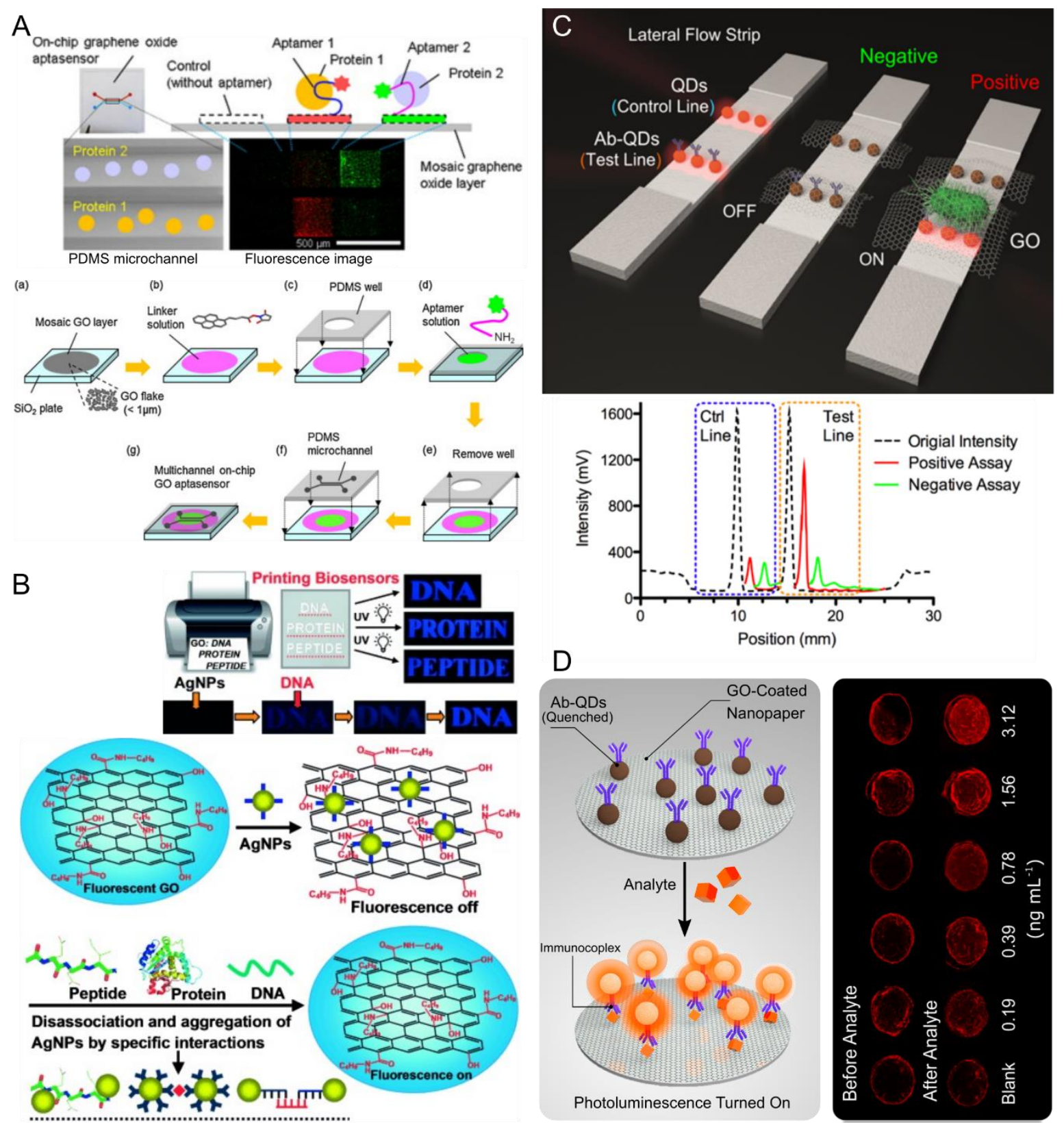

Figure 4. GO-based biosensing utilizing the solid phase. A. Aptamers anchored on GO substrates for protein detection in lab-on-a-chip format. Adapted with permission from ${ }^{[67]}$. Copyright 2015 Elsevier. B. Paper substrates printed with biosensing photloluminescent inks. Adapted with permission from ${ }^{[69]}$. Copyright 2012 John Wiley and Sons. C. Lateral flow device based on non-radiative energy transfer for pathogen detection. Adapted with permission from ${ }^{[73]}$. Copyright 2015 American Chemical Society. D. GO-coated nanopaper immunosensing platform for protein detection. Adapted with permission from ${ }^{[75]}$. Copyright 2017 John Wiley and Sons. 

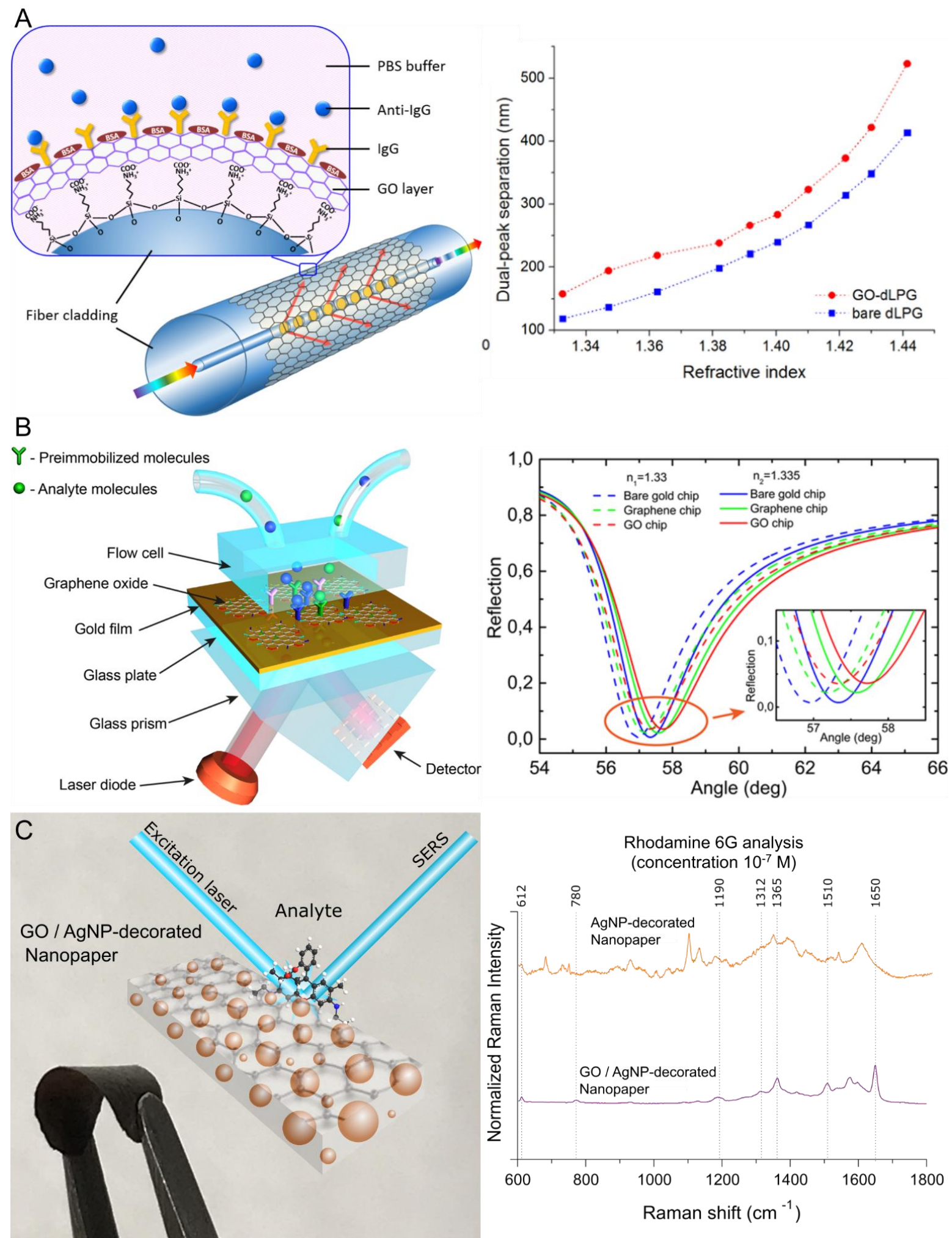

Figure 5. GO in optical label-free biosensing approaches and its respective advantageous contribution. A. Biosensing based on GO-coated optical fiber. Adapted with permission from ${ }^{[82]}$. Copyright 2017 Elsevier. B. Biosensing based on GO-coated SPR substrate. Adapted with permission from ${ }^{[88]}$. Copyright 2015 American Chemical Society. C. SERS substrateincorporating GO. Adapted with permission from ${ }^{[106]}$. Copyright 2018 John Wiley and Sons. 


\section{Author Photographs}

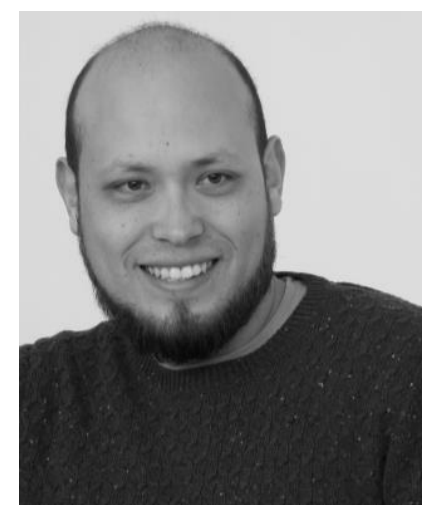

Eden Morales-Narváez is a Professor in the Department of Photonics and head of the Biophotonic Nanosensors Laboratory at the Center for Research in Optics, Mexico. He received a degree in Bionics Engineering from the National Polytechnic Institute (IPN) of Mexico in 2006 and the PhD in Biomedical Engineering from the Polytechnic University of Catalonia (UPC), Spain in 2013. He was a Postdoctoral Researcher at Nanobioelectronics and Biosensors Group (led by Prof. Arben Merkoçi) and started his independent career in late 2016. His research is focused on the development and application of micro/nanomaterialsbased sensing platforms for small molecules, proteins and cell analysis, particularly using graphene related-materials, quantum dots nanocrystals, nanocellulose and optical transduction systems at the cutting-edge.

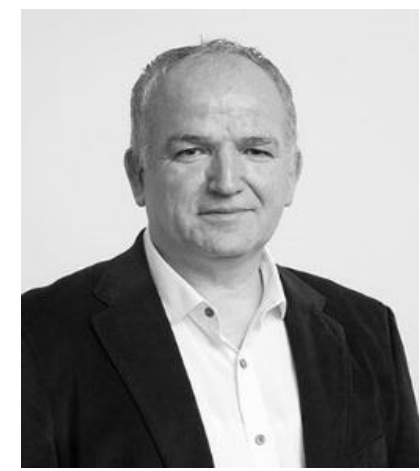

Arben Merkoçi is ICREA Professor and director of the Nanobioelectronics \& Biosensors Group at ICN2. After his PhD (1991) at Tirana University (Albania), in the topic of IonSelective-Electrodes (ISEs) Dr. Merkoçi worked as postdoc and senior researcher/invited professor in the field of nanobiosensors and lab-on-a-chip technologies in Italy, Spain, USA and since 2006 at ICN2. Prof. Merkoçi research is focused on the design and application of cutting edge nanotechnology and nanoscience based cost/efficient biosensors. These nanobiosensors involve integration of (bio)receptors with micro- and nanostructures/motors and applied in diagnostics, environmental monitoring or safety and security. He is co-founder of two spin-off companies, PaperDrop dedicated to nanodiagnostics and GraphenicaLab to electronic printing. 
Graphene oxide (GO) in optical biosensing. This paper highlights progressively discovered GO properties that influence the overall optical biosensing performance. From a critical point of view, new trends in GO-based optical biosensing are also discussed, along with research challenges, opportunities, and future perspectives in this topic.

\section{Biosensors}

E. Morales-Narváez*, A. Merkoçi*

Graphene Oxide as an Optical Biosensing Platform - a Progress Report

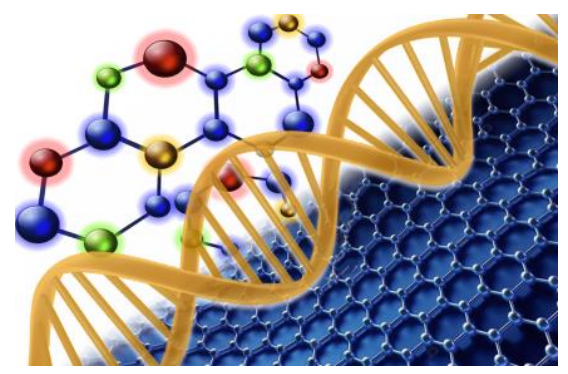

ToC figure 